\title{
REGULAR LINEAR ALGEBRAIC MONOIDS
}

BY

\author{
MOHAN S. PUTCHA ${ }^{1}$
}

\begin{abstract}
In this paper we study connected regular linear algebraic monoids. If $\phi$ : $G_{0} \rightarrow \mathrm{GL}(n, K)$ is a representation of a reductive group $G_{0}$, then the Zariski closure of $K \phi\left(G_{0}\right)$ in $\mathscr{M}_{n}(K)$ is a connected regular linear algebraic monoid with zero. In $\S 2$ we study abstract semigroup theoretic properties of a connected regular linear algebraic monoid with zero. We show that the principal right ideals form a relatively complemented lattice, that the idempotents satisfy a certain connectedness condition, and that these monoids are $V$-regular. In $\$ 3$ we show that when the ideals are linearly ordered, the group of units is nearly simple of type $A_{l}, B_{l}, C_{l}, F_{4}$ or $G_{2}$. In $\S 4$, conjugacy classes are studied by first reducing the problem to nilpotent elements. It is shown that the number of conjugacy classes of minimal nilpotent elements is always finite.
\end{abstract}

1. Preliminaries. Throughout this paper $Z^{+}$will denote the set of all positive integers. If $X$ is a set, then $|X|$ denotes the cardinality of $X$. Let $S$ be a semigroup. We let $E(S)$ denote the set of all idempotents of $S$. If $a, b \in S$, then $a \mid b$ (a divides $b)$ if $x a y=b$ for some $x, y \in S^{1}, a \mathscr{J} b$ if $a|b| a, a \mathscr{R} b$ if $a S^{1}=b S^{1}, a \mathscr{L} b$ if $S^{1} a=S^{1} b, \mathscr{H}=\mathscr{R} \cap \mathscr{L}, \mathscr{D}=\mathscr{R} \circ \mathscr{L}=\mathscr{L} \circ \mathscr{R}$ (see [1, 7]). The semigroups we consider turn out to have the property that a power of each element lies in a subgroup and hence by $[\mathbf{1 0}], \mathscr{J}=\mathscr{D}$. A $\mathscr{J}$-class with an idempotent is called regular. We let $\mathscr{U}(S)$ denote the partially ordered set of all regular $\mathscr{Z}$-classes of $S$. If $a \in S$, then $J_{a}, H_{a}$ will denote the $\mathscr{F}$ class and $\mathscr{H}$-class of $a$, respectively. If $X \subseteq S$, then $E(X)=E(S) \cap X$ and $\langle X\rangle$ is the subsemigroup of $S$ generated by $X$. $S$ is regular if $a \in a S a$ for all $a \in S$. A monoid $S$ with group of units $G$ is unit regular if for all $a \in S$ there exist $e \in E(S)$ and $x \in G$ such that $a=e x$. Clearly any submonoid of a unit regular monoid, containing the group of units, is also unit regular.

Let $K$ be an algebraically closed field, $K^{*}=K \backslash\{0\}$. We let $\mathscr{M}_{n}(K)$ denote the monoid of all $n \times n$ matrices over $K$. We consider $\mathscr{M}_{n}(K)$ with the Zariski topology on it (see [8]). By an algebraic monoid $S$ we mean a closed submonoid of $\mathscr{M}_{n}(K)$. The irreducible component of 1 in the Zariski topology of $S$ will be denoted by $S^{c}$. If $S=S^{c}$, then we say that $S$ is a connected monoid. Let $S$ be a connected monoid with zero and group of units $G$. Let $T$ be a maximal torus of $G$. Thus $E(\bar{T})$ consists of the 'diagonal' idempotents in $S$. Then $E(\bar{T})$ is a finite relatively complemented lattice

Received by the editors July 23, 1984 and, in revised form, December 3, 1984.

1980 Mathematics Subject Classification. Primary 20M10; Secondary 20 G99.

Key words and phrases. Matrix semigroups, algebraic groups, idempotents, nilpotents, conjugacy classes, regular semigroups.

${ }^{1}$ This research was partially supported by NSF Grant MCS8025597-A01.

(C1985 American Mathematical Society $0002-9947 / 85 \$ 1.00+\$ .25$ per page 
and $\mathscr{U}(S)$ is a finite lattice. Moreover $\operatorname{dim} T=$ the length of any maximal chain in $E(\bar{T})=$ the length of any maximal chain in $\mathscr{U}(S)=$ the length of any maximal chain in $E(S)$. We call this number the rank of $S$. Let $X, Y \subseteq S$. Then the centralizer in $Y$ of $X$ is $C_{Y}(X)=\{a \in Y \mid a x=x a$ for all $x \in X\}$ and the normalizer in $G$ of $X$ is $N_{G}(X)=\left\{a \in G \mid a^{-1} X a=X\right\}$. Let $\Gamma \subseteq E(\bar{T})$. Then the right centralizer of $\Gamma$ in $G$ is $C_{G}^{r}(\Gamma)=\{a \in G \mid a e=e a e$ for all $e \in \Gamma\}$. The left centralizer of $\Gamma$ in $G$ is $C_{G}^{l}(\Gamma)=\{a \in G \mid e a=e a e$ for all $e \in \Gamma\}$. The groups $C_{G}^{l}(\Gamma), C_{G}^{r}(\Gamma), C_{G}(\Gamma)$ are all connected by [18, Corollary 4.2]. Let $e \in E(S)$. Then $S_{e}=\{a \in S \mid e a=a e=e\}^{c}$, $G_{e}=G \cap S_{e}$ (see $[17,18]$ for further details). We refer to $[8,28]$ for the basic notions in the theory of algebraic groups. In particular, $W=N_{G}(T) / C_{G}(T)$ is the Weyl group of $G$.

Let $S$ be a connected algebraic monoid with zero and group of units $G$. If char $K=0$, then the author [21] showed that $G$ is a reductive group if and only if $S$ is a regular semigroup. This result was extended to arbitrary characteristic by Renner [24]. Renner uses some results in algebraic geometry such as Zariski's main theorem to prove this result. Because of the central importance of this result in the theory of linear algebraic monoids, we present here an alternate proof.

THEOREM $1.1[\mathbf{2 1}, \mathbf{2 4}]$. Let $S$ be a connected algebraic monoid with zero and group of units $G$. Then $G$ is a reductive group if and only if $S$ is a (unit) regular monoid.

Proof. If $S$ is a regular semigroup, then by [18, Theorem 2.11], $G$ is a reductive group. So assume that $G$ is a reductive group. We prove by induction on $\operatorname{dim} S$ that $S$ is regular. If $e \in E(S)$, then $G_{e}$ and $H_{e}$ are reductive groups by [21, Theorem 2.3]. Thus $e \neq 0$ implies that $S_{e}$ is regular, and $e \neq 1$ implies that $e S e$ is regular. Let $S \subseteq \mathscr{M}_{n}(K), \operatorname{dim} S=p$. Consider det: $S \rightarrow K$ where 'det' denotes the matrix determinant. Let $Y=S \backslash G=\operatorname{det}^{-1}(0)$. Then $Y=S Y S$. So by [8, Theorem 4.1], every irreducible component of $Y$ is an ideal of $S$ by dimension $p-1$. Let $Y_{0}$ be an irreducible component of $Y$. Then $\operatorname{dim} Y_{0}=p-1$. We first show that $Y_{0}$ is not nil. For suppose otherwise. Let $B, B^{-}$be opposite Borel subgroups of $G$ with respect to some maximal torus $T$. By [8, Proposition 27.2] we can choose an appropriate change of basis such that every element of $B$ is upper triangular and every element of $B^{-}$is lower triangular. Thus the same is true for $\bar{B}$ and $\bar{B}^{-}$. Thus every element of $\bar{B} \cap \bar{B}^{-}$ is a diagonal matrix. Let $W$ denote the Weyl group of $G$. So $B^{-}=\sigma^{-1} B \sigma$ for some $\sigma \in W$. Now $B^{-} B$ and hence $B \sigma B$ is an open subset of $G$ by [8, Proposition 28.5]. So $X=G \backslash B \sigma B$ is a closed subset of $G$. Thus $\operatorname{dim} X \leqslant p-1$. We claim that $Y_{0} \subseteq \bar{X}$. So let $a \in Y_{0}, a \notin \bar{X}$. Since $0 \in \bar{B} \subseteq \bar{X}, a \neq 0$. By the Bruhat decomposition [8, Theorem 28.3], $G$ is the disjoint union of $B \theta B(\theta \in W)$. Thus $a \notin \overline{B \theta B}$ for any $\theta \in W, \theta \neq \sigma$. So $a \notin \bar{B} B \theta B \cup B \theta B \bar{B}$ for $\theta \in W, \theta \neq \sigma$. Also by [29, p. 68 or 23, Proposition 3.4.1] $S=G \bar{B}=\bar{B} G$. Hence $a \in \bar{B} B \sigma B \cap B \sigma B \bar{B} \subseteq \bar{B} \sigma B \cap B \sigma \bar{B}$. Let $\sigma=g T, g \in N_{G}(T)$. Then there exist $b_{1}, b_{2} \in B, u_{1}, u_{2} \in \bar{B}$ such that $a=u_{1} g b_{1}=$ $b_{2} g u_{2}$. So $u_{2} b_{1}^{-1}=g^{-1} b_{2}^{-1} u_{1} g \in \bar{B} \cap \bar{B}^{-}$. Thus $u_{2} b_{1}^{-1}$ is a diagonal matrix. But $u_{2} b_{1}^{-1}=g^{-1} b_{2}^{-1} a b_{1}^{-1} \in Y_{0} \backslash\{0\}$. This contradicts the assumption that $Y_{0}$ is nil. Thus $Y_{0} \subseteq \bar{X}$. Let $X_{1}, \ldots, X_{r}$ be the irreducible components of $X_{i}$. Then $Y_{0} \subseteq \bar{X}_{i}$ for some $i$. Since $\operatorname{dim} Y_{0}=p-1$ and $\operatorname{dim} X_{i} \leqslant p-1$ we see that $Y_{0}=\bar{X}_{i}$. This is a contradiction since $Y_{0} \subseteq S \backslash G, X_{i} \subseteq G$. Therefore $Y_{0}$ is not nil. So there exists $e \in E\left(Y_{0}\right)$, 
$e \neq 0$. Choose $e$ such that $J_{e}$ is maximal in $\mathscr{U}\left(Y_{0}\right)$. Let $Y_{1}=\left\{y \in Y_{0} \mid y+e\right\}$. Then $Y_{1}$ is closed by [13, Lemma 2.1]. Let $u \in Y \backslash Y_{1}$. Then $u \mid e$. By [21, Theorem 1.4], $u \in G S_{e} G$. Hence $u$ is regular. By the maximality of $J_{e}, u \mathscr{J} e$. Thus $u \in S e S$. Hence $Y_{0}=\overline{S e S} \cup Y_{1}$. Since $e \notin Y_{1}$, and since $Y_{0}$ is irreducible, we see that $Y_{0}=\overline{S e S}$. Now $e \in \Gamma$ for some maximal chain in $E(S)$. Let $B_{1}=C_{G}^{r}(\Gamma), B_{2}=C_{G}^{l}(\Gamma)$. Then $B_{1}, B_{2}$ are Borel subgroups of $G$ by [18, Theorem 4.5]. Clearly, $B_{1} e=e B_{1} e$, $e B_{2}=e B_{2} e$. Thus $B_{1} e S e B_{2}=e S e$. So by [29, p. 68], GeSeG is closed in $S$. Since $G e G \subseteq G e S e G$, we see that $Y_{0}=\overline{S e S}=\overline{G e G} \subseteq G e S e G$. Since $e S e$ is regular, so is $Y_{0}$. Thus $Y=S \backslash G$ is regular. This proves the theorem.

Let $S$ be a connected regular monoid with zero and group of units $G$. Let $T$ be a maximal torus of $G$. If $\Gamma$ is a maximal chain in $E(\bar{T})$, then by $[\mathbf{1 8}] C_{G}^{r}(\Gamma)$ is a Borel subgroup of $G$. However, the map: $\Gamma \rightarrow C_{G}^{r}(\Gamma)$ is not one-to-one. To obtain a better correspondence, the author [18-20] considers cross-section lattices. A subset $\Lambda$ of $E(\bar{T})$ is a cross-section lattice if: (1) $|\Lambda \cap J|=1$ for all $J \in \mathscr{U}(S)$, and (2) for all $e, f \in \Lambda, J_{e} \geqslant J_{f}$ implies $e \geqslant f$. Thus a cross-section lattice is a diagonal idempotent cross-section of $\mathscr{J}$ classes preserving the $\mathscr{J}$ class ordering. The author [18-20] has shown that cross-section lattices correspond one-to-one with Borel subgroups of $G$ containing $T$. The correspondence is as follows:

$$
\begin{aligned}
\Lambda \rightarrow B & =C_{G}^{r}(\Lambda) \\
B \rightarrow \Lambda & =\{e \in E(\bar{T}) \mid a e=e a e \text { for all } a \in B\} \\
& =\{e \in E(\bar{T}) \mid \text { for all } f \in E(S), e \mathscr{R} f \text { implies } f \in \bar{B}\} .
\end{aligned}
$$

Let $C$ denote the center of the reductive group $G$. Then by $[\mathbf{8}] G=C G_{0}$, where $G_{0}$ is a semisimple group (the commutator subgroup of $G$ ). We will say that $G$ is nearly semisimple if $\operatorname{dim} C=1$. In such a case Renner $[25,26]$ calls the monoid $S$ semisimple. We avoid the terminology in this paper because the term 'semisimple' has a completely different meaning in abstract semigroup theory. With the additional assumption that $S$ is normal, Renner classifies these monoids geometrically. Let $X(T), X(\bar{T})$ denote the character group and character monoid of $T$ and $\bar{T}$, respectively. Let $\Phi$ denote the root system of $G$. Then Renner [26] shows that the map $S \rightarrow(X(T), \Phi, X(\bar{T}))$ classifies normal 'semisimple' monoids up to isomorphism. Since $X(T), \Phi, X(\bar{T})$ are all geometrical objects, this classification may be thought of as being geometrical. This theorem of Renner is quite deep, having many important consequences. For example, Renner uses his classification to show that any such monoid $S$ has an involution fixing $\bar{T}$. A key step in the proof of Renner's classification theorem is the generalization of Chevalley's big cell from groups to monoids. Renner uses cross-section lattices in the construction of his big cell.

Let $S$ be a connected regular monoid with zero and let $\hat{S}$ be its normalization (see [23]). Then there exists a finite homomorphism $\phi: \hat{S} \rightarrow S$. The term 'finite' is being used in the sense of algebraic geometry [8, §4.2]. Finite homomorphisms are of abstract semigroup theoretic interest also, because of the following result of Renner [23, Propositions 3.4.13, 4.1.6]. 
THEOREM 1.2 (RENNER). Let $\phi: S \rightarrow S^{\prime}$ be a homomorphism between connected regular monoids with zero such that $\overline{\phi(S)}=S^{\prime}$. Then $\phi$ is finite if and only if it is idempotent separating.

Following Renner [26] we say that a connected regular monoid $S$ with zero is Firreducible if $\mathscr{U}(S) \backslash\{0\}$ has a minimum element. The following result is due to Renner [26, Corollary 8.3.3].

THEOREM 1.3 (RENNER). Let $S$ be a connected regular monoid with zero. Then $S$ is Firreducible if and only if $S$ has a finite ( $i$. idempotent separating) irreducible matrix representation. In particular the group of units of such a monoid is nearly semisimple.

We will say that $G=C G_{0}$ is nearly simple if $\operatorname{dim} C=1$ and $G_{0}$ is almost simple, i.e. $G_{0}$ has no nontrivial normal closed connected subgroups. We denote by $\operatorname{Ren}(S)$ the finite inverse monoid $\overline{N_{G}(T)} / T$. Renner [27] has used this monoid to obtain a Bruhat decomposition for $S$. $\operatorname{Ren}(S)$ is a fundamental (i.e. has no nontrivial idempotent separating congruences) unit regular monoid with group of units $W=$ $N_{G}(T) / T$ and idempotent set $E(\bar{T})$.

2. Abstract properties. Let $S$ be a regular semigroup. We let $S / \mathscr{R}$ denote the partially ordered set of all principal right ideals of $S$ under inclusion. Then $S / \mathscr{R}$ can be identified with the partially ordered set of all $\mathscr{R}$-classes of $S$.

THEOREM 2.1. Let $S$ be a connected regular monoid with zero and group of units $G$. Then $S / \mathscr{R}$ is a relatively complemented, complete lattice with all maximal chains having the same finite length equal to rank $S$.

Proof. By [14] all maximal chains in $E(S)$ have the same finite length. Thus the same is true for $S / \mathscr{R}$. Let $e S, f S \in S / \mathscr{R}$ where $e, f \in E(S)$. By [18, Theorem 4.6] $C_{G}^{r}(e)$ and $C_{G}^{r}(f)$ are parabolic subgroups of $G$. So by [8, Corollary 28.3] $C_{G}^{r}(e) \cap$ $C_{G}^{r}(f)$ contains a maximal torus $T$ of $G$. By [14] there exist $a \in C_{G}^{r}(e), b \in C_{G}^{r}(f)$ such that $e^{\prime}=a^{-1} e a, f^{\prime}=b^{-1} f b \in \bar{T}$. Then $e S=e^{\prime} S, f S=f^{\prime} S, e^{\prime} f^{\prime}=f^{\prime} e^{\prime}$. So $e S \cap f S=e^{\prime} f^{\prime} S$. Since $S / \mathscr{R}$ has a maximum element, and since all maximal chains in $S / \mathscr{R}$ have the same finite length, it follows that $S / \mathscr{R}$ is a complete lattice.

Let $e, f, h \in E(S)$ such that $e S \supseteq f S \supseteq h S$. Without loss of generality we can assume that $e \geqslant f \geqslant h$. By [14] there exists a maximal torus $T$ of $G$ such that $e, f, h \in E(\bar{T})$. Since $E(\bar{T})$ is relatively complemented [13], there exists $f^{\prime} \in E(\bar{T})$ such that $e \geqslant f^{\prime} \geqslant h$ and $f f^{\prime}=h$. Then $e S \supseteq f^{\prime} S \supseteq h S$ and $f S \cap f^{\prime} S=h S$. It follows that $S / \mathscr{R}$ is relatively complemented.

Let $S$ be a regular semigroup, $a \in S$. Then $V(a)=\{x \in S \mid a x a=a, x a x=x\}$ denote the set of inverses of $a$ in $S$. Let $e, f \in E(S)$. Of crucial importance in the theory of regular semigroups is the sandwich set $\mathscr{S}(e, f)=V(e f) \cap f S e$ which is a rectangular band (see [11]).

THEOREM 2.2. Let $S$ be a connected regular monoid with zero and group of units $G$. Let $e, f \in E(S)$. Then

(i) $C_{G}^{r}(f) \cap C_{G}^{l}(e)$ is a connected group and $f S e \subseteq \overline{C_{G}^{r}(f) \cap C_{G}^{l}(e)}$.

(ii) If $h \in \mathscr{S}(e, f)$, then $\mathscr{S}(e, f)=\left\{x^{-1} h x \mid x \in C_{G}^{r}(f) \cap C_{G}^{l}(e)\right\}$. 
(iii) $\mathscr{S}(e, f)=\left\{e^{\prime} f^{\prime} \mid e^{\prime}, f^{\prime} \in E(\bar{T})\right.$ for some maximal torus $T$ of $G$ such that $\left.e \mathscr{L} e^{\prime}, f \mathscr{R} f^{\prime}\right\}=\left\{e^{\prime} f^{\prime} \mid e^{\prime}, f^{\prime} \in E(S), e \mathscr{L} e^{\prime}, f \mathscr{R} f^{\prime}, e^{\prime} f^{\prime}=f^{\prime} e^{\prime}\right\}$.

Proof. By [18, Theorem 4.6], $C_{G}^{r}(f), C_{G}^{l}(e)$ are parabolic subgroups of $G$. By [8, Corollary 28.3], there exists a maximal torus $T$ of $G$ contained in $C_{G}^{r}(f) \cap C_{G}^{l}(e)$. By [14], there exist $a \in C_{G}^{l}(e), b \in C_{G}^{r}(f)$ such that $e^{\prime}=a^{-1} e a, f^{\prime}=b^{-1} f b \in E(\bar{T})$. Then $f \mathscr{R} f^{\prime}, e \mathscr{L} e^{\prime}$. Hence by [15, Theorems 1, 9], $C_{G}^{l}(e)=C_{G}^{l}\left(e^{\prime}\right), C_{G}^{r}(f)=C_{G}^{r}\left(f^{\prime}\right)$. Since $f^{\prime} \in \bar{T} \subseteq \overline{C_{G}^{l}\left(e^{\prime}\right)}$, it follows from [18, Theorem 4.1] that $G^{\prime}=C_{G}^{l}(e) \cap C_{G}^{r}(f)$ is a connected group. Let $S^{\prime}=\bar{G}^{\prime}, S_{1}=\overline{C_{G}^{l}\left(e^{\prime}\right)}$. By [15, Theorem 1] $S e^{\prime} \subseteq S_{1}$. By the same theorem, $f^{\prime} S_{1} \subseteq S^{\prime}$. Clearly $f^{\prime} S e^{\prime} \subseteq f^{\prime} S_{1}$ and $f S e=f^{\prime} S e^{\prime}$. Thus $f S e \subseteq S^{\prime}$. Since $e^{\prime} f^{\prime}=f^{\prime} e^{\prime}$, we see that $e^{\prime} f^{\prime} \in \mathscr{S}(e, f)$. Let $x \in G^{\prime}$. Then $x^{-1} e^{\prime} f^{\prime} x=e^{\prime \prime} f^{\prime \prime}$ where $e^{\prime \prime}=x^{-1} e^{\prime} x, f^{\prime \prime}=x^{-1} f^{\prime} x \in \overline{x^{-1} T x}$, e $\mathscr{L} e^{\prime \prime}, f \mathscr{R} f^{\prime \prime}$. Hence $e^{\prime \prime} f^{\prime \prime} \in \mathscr{S}(e, f)$. Finally let $h \in \mathscr{S}(e, f)$. Since $\mathscr{S}(e, f)$ is a rectangular band, and since $\mathscr{S}(e, f) \subseteq$ $f S e \subseteq S^{\prime}$, we see that $h \mathscr{J} e^{\prime} f^{\prime}$ in $S^{\prime}$. By [15, Theorem 9], there exists $x \in G^{\prime}$ such that $h=x^{-1} e^{\prime} f^{\prime} x$. This proves the theorem.

COROLLARY 2.3. Let $S$ be a connected regular monoid with zero and group of units $G$. Let $a \in S$. Then there exists a maximal torus $T$ of $G, e, f \in E(\bar{T})$ such that $e \mathscr{R}$ a $\mathscr{L} f$. In particular the number of conjugacy classes of the $\mathscr{H}$-classes of $S$ is finite.

Let $S$ be a regular semigroup. Then $S$ is $V$-regular if $V(a b) \subseteq V(b) V(a)$ for all $a, b \in S . S$ is strongly V-regular if for all $e, f \in E(S), h \in \mathscr{S}(e, f)$, there exist $e^{\prime}, f^{\prime} \in E(S)$ such that $e \mathscr{L} e^{\prime}, f \mathscr{R} f^{\prime}, h \in \mathscr{S}\left(f^{\prime}, e^{\prime}\right)$. Nambooripad and Pastijn [12] show that every strongly $V$-regular semigroup is $V$-regular. They also show that many classes of naturally arising regular semigroups are strongly $V$-regular. We add to this list. The following theorem is immediate from Theorem 2.2.

THEOREM 2.4. Every connected regular monoid with zero is strongly V-regular.

Let $S$ be a regular semigroup. Then Fitz-Gerald [5] showed that $\langle E(S)\rangle$ is a regular semigroup. The determination of $\langle E(S)\rangle$ represents an important step in the study of $S$ (see $[6,11])$.

Problem 2.5. Let $S$ be a connected regular monoid with zero. Determine $\langle E(S)\rangle$.

We solve the above problem in the special case when $S \backslash G$ has a maximum $\mathscr{F}$-class. Erdos [4] (see also Dawlings [2, 3]) showed that the singular matrices in $\mathscr{M}_{n}(K)$ can always be written as products of idempotent matrices.

LEMMA 2.6. Let $S$ be a connected regular monoid with zero, $S_{1}$ a closed connected submonoid of $S$. If $E(S)=E\left(S_{1}\right)$, then $S=S_{1}$.

Proof. We prove the lemma by induction on $\operatorname{dim} S$. Let $S \subseteq \mathscr{M}_{n}(K)$. Consider det: $S \rightarrow K$ where 'det' denotes the determinant. Let $V$ be an irreducible component of $\operatorname{det}^{-1}(0)$. Then $V$ is clearly an ideal of $S$ and by [8, Theorem 4.1], $\operatorname{dim} V=\operatorname{dim} S$ -1 . Since $S$ is regular, $V=S e S$ for some $e \in E(S), e \neq 1$. Now $e S_{1} e \subseteq e S e$, $E\left(e S_{1} e\right)=E(e S e)$, and $e S e$ is regular. By the induction hypothesis, $e S e=e S_{1} e$. Let $X=\{f \in E(S) \mid e \mathscr{R} f\}, Y=\{f \in E(S) \mid e \mathscr{L} f\}$. By [15, Theorem 14], $\operatorname{dim} \overline{S_{1} e S_{1}}=$ $\operatorname{dim} X+\operatorname{dim} Y+\operatorname{dim} e S_{1} e, \operatorname{dim} S e S=\operatorname{dim} X+\operatorname{dim} Y+\operatorname{dim} e S e . T$ Thus $\operatorname{dim} S e S$ $=\operatorname{dim} S_{1} e S_{1}<\operatorname{dim} S_{1}$. So $\operatorname{dim} S \leqslant \operatorname{dim} S_{1}$ and $S=S_{1}$. 
THEOREM 2.7. Let $S$ be a connected regular monoid with zero and group of units $G$. Suppose $S \backslash G$ has a maximum ffclass. Then $S \backslash G \subseteq E(S)^{m}=\langle E(S)\rangle$ for some $m \in Z^{+}$.

Proof. Let $J$ denote the maximum $\mathscr{F}$ class of $S \backslash G, E_{0}=E(S) \cap J$. Then $E_{0}$ is closed and irreducible by [15, Theorem 8$]$. We have the product maps $E_{0} \times \cdots \times$ $E_{0} \rightarrow \overline{E_{0}^{k}}$. Thus each $\overline{E_{0}^{k}}$ is also irreducible. Clearly

$$
E_{0} \subseteq \overline{E_{0}^{2}} \subseteq \overline{E_{0}^{3}} \subseteq \cdots
$$

So there exists $p \in Z^{+}, p \leqslant \operatorname{dim} S$ such that $\overline{E_{0}^{p}}=\overline{E_{0}^{q}}$ for all $q \in Z^{+}, q \geqslant p$. Let $S_{1}=\overline{E_{0}^{p}}$. Since $E(\bar{T})$ is relatively complemented for any maximal torus $T$ of $G$, it follows that $E(S) \backslash\{1\} \subseteq\left\langle E_{0}\right\rangle$. Thus $E\left(S_{1}\right)=E(S) \backslash\{1\}$. Let $e \in E_{0}$. Then $E\left(e S_{1} e\right)=E(e S e)$. By Lemma 2.6, $e S e=e S_{1} e$. Let $X=E_{0} \times \cdots \times E_{0}$, the $p$-fold direct product. Define $\phi: X \rightarrow e S e$ as $\phi\left(e_{1}, \ldots, e_{p}\right)=e e_{1} \cdots e_{p} e$. Thus $\phi(X)=$ eEge. So

$$
\overline{\phi(X)}=\overline{e E_{0}^{p} e}=e S_{1} e=e S e .
$$

By [8, Theorem 4.4], $\phi(X)$ is constructible and hence contains a nonempty open subset $U$ of $e S e$. Let $H$ denote the group of units of $e S e$. Then $H$ is an open subset of $e S e$. Thus $U_{1}=U \cap H$ is a nonempty open subset of $H$. By [8, Lemma 7.4], $U_{1}^{2}=H$. Thus $H \subseteq E_{0}^{2 p+3}$.

Now let $a \in J$. Then there exist $e, f \in E_{0}$ such that $e \mathscr{R} a \mathscr{L} f$. By [16, Lemma 1.12] there exist $e_{1}, f_{1} \in E_{0}$ such that $e \mathscr{R} e_{1} \mathscr{L} f_{1} \mathscr{R} f$. Then $e_{1} f \mathscr{H} a$. So $a \in H_{a}=$ $H_{e} e_{1} f \subseteq E_{0}^{2 p+5}$. Finally, let $a \in S \backslash G$. Then $a \mathscr{R} f$ for some $f \in E(S), f \neq 1$. Now $f^{\prime} \geqslant f$ for some $f^{\prime} \in E_{0}$ (see [15]). By [15], $a=f g$ for some $g \in G$. So $a=f\left(f^{\prime} g\right)$, $f^{\prime} g \in J \subseteq E_{0}^{2 p+5}$. Thus $a \in E_{0}^{2 p+6}$. This proves the theorem.

EXAmple 2.8. Let $S=\left\{A \otimes B \mid A, B \in \mathscr{M}_{2}(K)\right\}$. Then for $B \in \mathrm{GL}(2, K)$, $\left(\begin{array}{ll}1 & 0 \\ 0 & 0\end{array}\right) \otimes B$ cannot be written as a product of idempotents in $S$ unless $B$ is a scalar.

EXAMPLE 2.9. Let $S=\overline{K G_{0}}$ where $G_{0}=\left\{A \otimes\left(A^{-1}\right)^{t} \mid A \in \operatorname{SL}(3, K)\right\}$. Then $\mathscr{U}(S)$ is the same as in Example 2.8. However $S \backslash G$ is generated by idempotents where $G=K^{*} G_{0}$. Note that $S \backslash G$ has two maximal $\mathbb{Z}$ classes.

Problem 2.10. Let $S$ be a connected regular monoid with zero and group of units $G$. Find necessary and sufficient conditions for $S \backslash G$ to be idempotent generated.

Let $S$ be a regular semigroup. The fundamental congruence $\mu$ on $S$ is the largest congruence on $S$ contained in $\mathscr{H}$. If $\mu$ equals the equality, then $S$ is said to be fundamental (see $[6,7,11])$. We now show that when $S$ is a connected regular monoid with zero, then the extension $S$ of $S / \mu$ is a special 'central' extension and hence susceptible to a cohomological approach [9].

THEOREM 2.11. Let $S$ be a connected regular monoid with zero and group of units $G$. Consider the natural (semigroup) homomorphism $\phi: S \rightarrow S / \mu$. Then for all $e \in E(S)$, $\phi^{-1} \phi(e)$ is the center of $H_{e}$.

Proof. Let $e \in E(S)$. Then by Hall [6, Corollary 6], $\mu$ restricted to $e S e$ is the fundamental congruence on $e S e$. Thus without loss of generality we can assume that 
$e=1$. Let $C$ denote the center of $G$. Let $x \in G$ such that $x \mu 1$. Then for all $f \in E(S), f x \mathscr{H} f \mathscr{H} x f$. So $f x=x f$ for all $f \in E(S)$. By [18, Theorem 4.5], $x \in B$ for all Borel subgroups $B$ of $G$. It follows [8, p. 162, Exercise 2] that $x \in C$. Now the congruence $\theta$ defined by $a \theta b$ if $a \in C b$, is idempotent separating and hence $\theta \subseteq \mu$. Thus $c \mu 1$ for all $c \in C$.

Since all maximal chains in $E(S)$ have the same finite length, we can define an obvious height function ht on $E(S)$ such that $h t(1)=\operatorname{rank} S$ and $h \mathrm{t}(0)=0$.

THEOREM 2.12. Let $S$ be a connected regular monoid with zero and group of units $G$. Let $e, e^{\prime} \in E(S)$ with $\mathrm{ht}(e)=\mathrm{ht}\left(e^{\prime}\right)=p>0$. Then there exist $e=e_{0}, e_{1}, \ldots, e_{k}=e^{\prime}$, $f_{1}, \ldots, f_{k} \in E(S)$ such that $\mathrm{ht}\left(e_{i}\right)=p, \operatorname{ht}\left(f_{i}\right)=p-1, e_{i}>f_{i}, e_{i-1}>f_{i}, i=1, \ldots, k$.

Proof. By [16, Lemma 1.15], the theorem is true when $G$ is a torus. Since any idempotent in $S$ is in the closure of a maximal torus and since any two maximal tori are conjugate, we are reduced to the case when $e \mathscr{J} e^{\prime}$. By [16, Lemma 1.12] we are then further reduced to the case when $e \mathscr{R} e^{\prime}$. There exists a cross-section lattice $\Lambda$ of $S$ such that $e \in \Lambda$. Let $B=C_{G}^{r}(\Lambda), T=C_{G}(\Lambda)$. Then $B$ is a Borel subgroup of $G, T$ is a maximal torus of $G, T \subseteq B, e \in E(\bar{T}), e^{\prime} \in \bar{B}$. By [20, Theorem 1.2], there exists a cross-section lattice $\Lambda^{\prime} \subseteq E(\bar{T})$ such that $B=C_{G}^{l}\left(\Lambda^{\prime}\right)$. There exists $u \in \Lambda^{\prime}$ such that $e \mathscr{J} u$ in $S$. Then $\operatorname{ht}(e)=\operatorname{ht}(u)$. So there exist $e=u_{0}, u_{1}, \ldots, u_{k}=u, v_{1}, \ldots, v_{k}$ $\in E(\bar{T})$ such that $\mathrm{ht}\left(u_{i}\right)=p, \operatorname{ht}\left(v_{i}\right)=p-1, u_{i}>v_{i}, u_{i-1}>v_{i}, i=1, \ldots, k$. By $[18$, Proposition 4.3], $e, e^{\prime}, v_{1} \in \overline{C_{B}^{r}\left(e, v_{1}\right)}$. There exists $x \in C_{B}^{r}\left(e, v_{1}\right)$ such that $e^{\prime}=$ $x^{-1}$ ex. Let $v_{1}^{\prime}=x^{-1} v_{1} x \in E(\bar{B})$. Then $v_{1} \mathscr{R} v_{1}^{\prime}, e^{\prime} \geqslant v_{1}^{\prime}$. Now $v_{1}, v_{1}^{\prime}, u_{2} \in \overline{C_{B}^{r}\left(u_{2}, v_{1}\right)}$. There exists $y \in C_{B}^{r}\left(u_{2}, v_{1}\right)$ such that $v_{1}^{\prime}=y^{-1} v_{1} y$. Let $u_{2}^{\prime}=y^{-1} u_{2} y \in E(\bar{B})$. Then $u_{2}^{\prime} \geqslant v_{1}^{\prime}, u_{2} \mathscr{R} u_{2}^{\prime}$. Continuing, we find $e^{\prime}=u_{0}^{\prime}, u_{1}^{\prime}, \ldots, u_{k}^{\prime}=u^{\prime}, v_{1}^{\prime}, \ldots, v_{k}^{\prime} \in E(\bar{B})$ such that $\operatorname{ht}\left(u_{i}^{\prime}\right)=p, \operatorname{ht}\left(v_{i}^{\prime}\right)=p-1, u_{i}^{\prime}>v_{i}^{\prime}, u_{i-1}^{\prime}>v_{i}^{\prime}, u_{i} \mathscr{R} u_{i}^{\prime}, v_{i} \mathscr{R} v_{i}^{\prime}, i=1, \ldots, k$. In particular, $u \mathscr{R} u^{\prime}$ and $u^{\prime}=z u z^{-1}$ for some $z \in C_{B}^{r}(u)$. Since $B \subseteq C_{G}^{l}(u)$, we see that $u=u^{\prime}$. This proves the theorem.

3. $\mathscr{J}$-linear monoids. The monoid $\mathscr{M}_{n}(K)$ has the very nice property that the $\mathscr{F}$ classes are linearly ordered. We will call such a monoid $\mathscr{F}$ linear. We start by obtaining a useful test for $\mathscr{Z}$ linearity.

THEOREM 3.1. Let $S$ be a connected regular monoid with zero and group of units $G$. Let rank $S=m$. Then the following conditions are equivalent.

(i) $S$ if $\mathscr{F}$ linear.

(ii) There exists $a \in S$ such that $a^{m}=0, a^{m-1} \neq 0$.

(iii) There exists $a \in \operatorname{Ren}(S)$ such that $a^{m}=0, a^{m-1} \neq 0$.

Proof. We first show that (ii) $\Rightarrow$ (i). We proceed by induction on $\operatorname{dim} S$. If $\operatorname{dim} S=1$, then $|\mathscr{U}(S)|=2$ and hence $S$ is $\mathscr{F}$ linear. So let $\operatorname{dim} S>1$. Let $a \in S$ such that $a^{m}=0, a^{m-1} \neq 0$. Let $J_{i}$ denote the $\mathscr{f}$ class of $a^{i}$. Then $J_{i} \neq J_{k}$ for $1 \leqslant i<k \leqslant m$ (recall that $S$ is a matrix semigroup). Since $S$ is regular, each $J_{i}$ is regular. Thus $\left\{G>J_{1}>J_{2}>\cdots>J_{m}=0\right\}$ is a maximal chain in $\mathscr{U}(S)$. By Corollary 2.3, there exists a maximal torus $T$ of $G, e, f \in E(\bar{T})$ such that $e \mathscr{R}$ a $\mathscr{L} f$. So there exist $x, y \in G$ such that $a=e x=y f$. Now rank $e S e=m-1$, $(e x e)^{m-1}=$ $0,(e x e)^{m-2} \neq 0$. By the induction hypothesis, $e S e$ is $\mathscr{F}$ linear. Hence by [17, §4], the 
interval $\left[0, J_{1}\right]=\left\{0, J_{m-1}, \ldots, J_{1}\right\}$. Now $a^{2}=y f e x \mathscr{J}$ ef. So $e f=f e \in J_{2}, e \neq f$. By [16, Proposition 1.14(6)], the interval $\left[J_{2}, G\right]=\left\{J_{2}, J_{1}, G\right\}$. It then follows from [16, Lemma 1.15] that $\mathscr{U}(S)=\left\{0, J_{m-1}, \ldots, J_{1}, G\right\}$. Hence $S$ is $\mathscr{J}$-linear.

That (iii) $\Rightarrow$ (ii) is obvious. We are therefore left with showing (i) $\Rightarrow$ (iii). So assume that $S$ is $\mathscr{J}$ linear. Let $T$ be a maximal torus of $G$. If $e \in E(\bar{T}), \sigma \in W$, then let $e^{\sigma}=\sigma^{-1} e \sigma$. Let $\left\{1=e_{0}>e_{1}>\cdots>e_{m}=0\right\}$ be a maximal chain in $E(\bar{T})$. Since $E(\bar{T})$ is relatively complemented, there exist $1=f_{0}, f_{1}, \ldots, f_{m}=0 \in E(\bar{T})$ such that $e_{i+1} \leqslant f_{i} \leqslant f_{i-1}, e_{i} f_{i}=e_{i+1}, i=1, \ldots, m-1$. Since $S$ is $\mathscr{J}$-linear, $e_{i} \mathscr{J} f_{i}$, $i=1, \ldots, m-1$. Thus by $[17, \S 4]$, there exist $\sigma_{i} \in W, i=1, \ldots, m-1$, such that $e_{i}^{\sigma_{i}}=f_{i}, f_{j}^{\sigma_{i}}=f_{j}$ for $j<i, e_{k}^{\sigma_{i}}=e_{k}$ for $k>i$. Let $\sigma=\sigma_{1} \cdots \sigma_{m-1}$. Then

$$
e_{i}^{\sigma}=\left(e_{i}^{\sigma_{1} \cdots \sigma_{i-1}}\right)^{\sigma_{i} \cdots \sigma_{m-1}}=e_{i}^{\sigma_{i} \cdots \sigma_{m-1}}=f_{i}^{\sigma_{i+1} \cdots \sigma_{m-1}}=f_{i}, \quad i=1, \ldots, m-1 .
$$

So $e_{i} e_{i}^{\sigma}=e_{i+1}, i=1, \ldots, m-1$. Thus

$$
e_{1} e_{1}^{\sigma}=e_{2}, e_{1} e_{1}^{\sigma} e_{1}^{\sigma^{2}}=\left(e_{1} e_{1}^{\sigma}\right)\left(e_{1} e_{1}^{\sigma}\right)^{\sigma}=e_{2} e_{2}^{\sigma}=e_{3}, \ldots, e_{1} e_{1}^{\sigma} e_{1}^{\sigma^{2}} \cdots e_{1}^{\sigma^{k}}=e_{k+1}, \ldots
$$

Now $e_{1} e_{1}^{\sigma} \cdots e_{1}^{\sigma^{k}}=\left(e_{1} \sigma^{-1}\right)^{k+1} \sigma^{k+1}$. Thus $\left(e_{1} \sigma^{-1}\right)^{m}=0, \quad\left(e_{1} \sigma^{-1}\right)^{m-1} \neq 0$. This proves the theorem.

REMARK 3.2. Let $G_{0}$ be an almost simple group of type $A_{l}, B_{l}$ or $C_{l}$ with the representation given in Humphreys $[\mathbf{8}, \S 7.2]$. Let $S=\overline{K G_{0}}$. Then Theorem 3.1 can be used to show that $S$ is $\mathscr{J}$-linear. The $\mathscr{F}$ linearity of $S$ does indeed very much depend on the particular representation of $G_{0}$. For example, let $G_{0}=\left\{A \otimes\left(A^{-1}\right)^{t} \mid A \in\right.$ $\mathrm{SL}(3, K)\}$. Then $S=\overline{K G_{0}}$ is certainly not $\mathscr{F}$ linear.

THEOREM 3.3. Let $S$ be a connected regular Flinear monoid with zero and group of units $G$. Then $G$ is a nearly simple group of type $A_{l}, B_{l}, C_{l}, F_{4}$ or $G_{2}$.

Proof. Let $R$ denote the radical of $G$. Then $R$ lies in the center of $G$ and $G=R G_{0}$ where $G_{0}$ is a semisimple group. By Theorem $1.3, \operatorname{dim} R=1$. Suppose that $G_{0}$ is not almost simple. Then by [8, Theorem 27.5], $G_{0}=G_{1} G_{2}$ where $G_{1}, G_{2}$ are nontrivial semisimple subgroups of $G_{0}$ and $G_{1}$ centralizes $G_{2}$. Let $T$ be a maximal torus of $G$. Then $T_{1}=T \cap G_{1}, T_{2}=T \cap G_{2}$ are maximal tori of $G_{1}$ and $G_{2}$, respectively. Let $e_{i}$ be a nonzero minimal idempotent of $\overline{R T}_{i}, i=1,2$. Let $J_{i}$ denote the $\mathscr{Z}_{\text {class }}$ of $e_{i}$ in $S$, $i=1,2$. By symmetry suppose that $J_{1} \geqslant J_{2}$. Then $e_{1} \geqslant e_{2}^{\prime}$ for some $e_{2}^{\prime} \in E\left(J_{2}\right)$. There exists $x \in G$ such that $x^{-1} e_{2} x=e_{2}^{\prime}$. Now $x=x_{1} x_{2}$ for some $x_{1} \in G_{1}$, $x_{2} \in R G_{2}$. Since $e_{2} \in S_{2}=\overline{R G_{2}}$ and $G_{1}$ centralizes $S_{2}$, we see that $e_{2}^{\prime}=x_{2}^{-1} e_{2} x \in S_{2}$. Now since $R G_{1}$ is nearly semisimple, we see by [18, Theorems $\left.2.3,2.13\right]$ that there exists $e_{1}^{\prime} \in E\left(\overline{R T_{1}}\right), e_{1} \neq e_{1}^{\prime}$ such that $e_{1} \mathscr{J} e_{1}^{\prime}$ in $S_{1}=\overline{R G_{1}}$. Thus $e_{1}^{\prime}=y^{-1} e_{1} y$ for some $y \in G_{1}$. Then $e_{1}^{\prime} \geqslant y^{-1} e_{2}^{\prime} y=e_{2}^{\prime}$. So $0=e_{1} e_{1}^{\prime} \geqslant e_{2}^{\prime}$, a contradiction. This shows that $G$ is nearly simple.

Let $T$ be a maximal torus of $G$ and let $\Lambda=\left\{0<e_{1}<\cdots<e_{k}<1\right\}$ be a maximal chain in $E(\bar{T})$. Then $\Lambda$ is a cross-section lattice. Let $B=C_{G}^{r}(\Lambda)$. For $i=1, \ldots, k$, there exists $\sigma_{i} \in W, \sigma_{i}^{2}=1$ such that $e_{i}^{\sigma_{i}} \neq e_{i}, e_{j}^{\sigma_{i}}=e_{j}$ for $j \neq i$. By [20, Corollary 2.8], $\mathscr{F}=\left\{\sigma_{i} \mid i=1, \ldots, k\right\}$ is the set of simple reflections with respect to $B$. Let $\mathscr{F}_{i}=\mathscr{F} \backslash\left\{\sigma_{i}\right\}, P_{i}=C_{G}^{r}\left(e_{i}\right) \supseteq B$. Then $W_{i}=\left\langle\mathscr{F}_{i}\right\rangle$ is the Weyl group of $P_{i}$ and hence $P_{i}=B W_{i} B$. Clearly $C_{G}\left(e_{i}\right)$ is a Levi factor of $P_{i}$. Let $H_{i}$ denote the $\mathscr{H}$-class of 
$e_{i}, G_{i}=G_{e_{i}}$. Then $H_{i}, G_{i}$ are the groups of units of $e_{i} S e_{i}$ and $S_{e_{i}}$, respectively. By [17, Theorem 4.6], $e_{i} S e_{i}, S_{e_{i}}$ are $\mathscr{Z}$ linear monoids. Thus $H_{i}, G_{i}$ are nearly simple groups. By [15, Theorem 4], the homomorphism $\psi: C_{G}\left(e_{i}\right) \rightarrow H_{i}$ given by $\psi(a)=e_{i} a$ is surjective. Clearly $G_{i}$ is the identity component of the kernel of $\psi$. It follows that for $i \neq 1, k, W_{i}$ is reducible. Hence $G$ is not of type $D_{l}, E_{6}, E_{7}$ or $E_{8}$. This proves the theorem.

THEOREM 3.4. Let $S$ be a connected regular Flinear monoid with zero and group of units $G$. Let $T$ be a maximal torus of $G$. Then:

(i) Cross-section lattices are just the maximal chains in $E(\bar{T})$.

(ii) The Weyl group of $G$ is isomorphic to the abstract automorphism group of $E(\bar{T})$.

(iii) $\operatorname{Ren}(S)$ is isomorphic to the Munn semigroup of $E(\bar{T})$.

Proof. (i) is clear and (ii) follows from [18, Theorem 3.9]. We now prove (iii). The fundamental congruence $\mu$ on the inverse monoid $\overline{N_{G}(T)}$ is given by $a \mu b$ if and only if $b \in T a$. Thus $\operatorname{Ren}(S)$ is isomorphic to the submonoid of the Munn semigroup of $E(\bar{T})$ generated by $W$ and $E(\bar{T})$. It therefore suffices to show that any isomorphism between two principal ideals of $E(\bar{T})$ extends to an automorphism of $E(\bar{T})$. Let $\phi: e E(\bar{T}) \cong f E(\bar{T})$ where $e, f \in E(\bar{T})$. Then $e \mathscr{J} f$ and so $\sigma^{-1} e \sigma=f$ for some $\sigma \in W$. Thus we are reduced to the case when $e=f$. Then $\phi$ is an automorphism of $e E(\bar{T})=E(\overline{e T})$ and $e S e$ is $\mathscr{J}$ linear. So by (ii) $\phi$ belongs to the Weyl group $e W$ of $e S e$. Hence $\phi$ extends to an automorphism of $E(\bar{T})$. This proves the theorem.

4. Conjugacy classes. We start with the following preliminary result.

THEOREM 4.1. Let $S$ be a connected regular monoid with zero and group of units $G$, rank $S=m$. Let $e \in E(S)$. Let $N_{e}$ denote the set of nilpotent elements of $S_{e}$ and let $G^{e}=C_{G}\left(S_{e}\right)^{c}$. Let $a, b \in S$ such that $a^{m} \mathscr{H}$ e $\mathscr{H} b^{m}$. Then there exist $\eta \in N_{e}, g \in G^{e}$ such that $a=\eta g$. Moreover $a$ is conjugate to $b$ if and only if $b=\eta^{\prime} g^{\prime}$ for some $\eta^{\prime} \in N_{e}$, $g^{\prime} \in G^{e}$ such that $\eta$ is conjugate to $\eta^{\prime}$ in $S_{e}$ and $g$ is conjugate to $g^{\prime}$ in $G^{e}$.

Proof. Now $G_{e} \triangleleft C_{G}(e)$ and $C_{G}(e)$ is a reductive group. Thus by [8, Theorem 27.5], $C_{G}(e)=G_{e} G^{e}$. Now $e a \in S a^{m+1} \subseteq S e, a e \in a^{m+1} S \subseteq e S$. So $e a=a e \mathscr{H} e$. By [15, Theorem 4] there exists $x \in G^{e}$ such that $e a=e x$. So $e a x^{-1}=a x^{-1} e=e$. Thus $a x^{-1} \in S_{e}^{\prime}=\{z \in S \mid e z=z e=e\}$. Let $G_{e}^{\prime}=G \cap S_{e}^{\prime}$. Then by [21, Theorem 1.3], $S_{e}^{\prime}=G_{e}^{\prime}$. By [21, Lemma 1.2], $S_{e}^{\prime}=S_{e} G_{e}^{\prime} \subseteq S_{e} C_{G}(e)=S_{e} G_{e} G^{e}=S_{e} G^{e}$. So there exists $\eta \in S_{e}, g_{1} \in G^{e}$ such that $a x^{-1}=\eta g_{1}$. Let $g=g_{1} x \in G^{e}$. Then $a=\eta g$. Now $\eta^{m} \mathscr{H} f$ for some $f \in E\left(S_{e}\right)$. So $f \geqslant e$. Now e $\mathscr{H} a^{m}=(\eta g)^{m}=\eta^{m} g^{m} \mathscr{J} \eta^{m} \mathscr{H} f$. Thus $e \mathscr{J} f, f \geqslant e$. Hence $e=f$. Since $\eta \in S_{e}, \eta^{m}=e$. Thus $\eta \in N_{e}$. Now suppose $a$ is conjugate to $b$. Then $y^{-1} a y=b$ for some $y \in G$. Then $e \mathscr{H} b^{m}=y^{-1} a^{m} y \mathscr{H} y^{-1} e y$. So $y^{-1} e y=e$ and $y \in C_{G}(e)$. Hence $y=y_{1} y_{2}$ for some $y_{1} \in G_{e}$ and $y_{2} \in G^{e}$. Then $b=\eta^{\prime} g^{\prime}$ where $\eta^{\prime}=y^{-1} \eta y=y_{1}^{-1} \eta y_{1}, g^{\prime}=y^{-1} g y=y_{2}^{-1} g_{2} y_{2}$. Conversely let $b=\eta^{\prime} g^{\prime}$, $\eta^{\prime} \in N_{e}, g^{\prime} \in G^{e}$, such that $\eta^{\prime}=y_{1}^{-1} \eta y_{1}$ for some $y_{1} \in G_{e}$ and $g^{\prime}=y_{2}^{-1} g y_{2}$ for some $y_{2} \in G^{e}$. Then $\eta^{\prime}=y^{-1} \eta y, g^{\prime}=y^{-1} g y$ where $y=y_{1} y_{2}$. So $y^{-1} a y=b$. This proves the theorem.

Let $S$ be a connected regular monoid with zero and group of units $G$, rank $S=m$. Let $a, b \in S$. Then for some $e, f \in E(S), a^{m} \mathscr{H} e, b^{m} \mathscr{H} f$. If $a$ is conjugate to $b$ 
then $e$ is conjugate to $f$. If $e=f$, then by Theorem 4.1, the conjugacy problem for $a$ and $b$ reduces to that for nilpotent elements in $S_{e}$ and that for elements in the reductive group $G^{e}$. Thus the conjugacy problem in $S$ reduces to the following three problems:

(A) Conjugacy problem for idempotents.

(B) Conjugacy problem within a reductive group.

(C) Conjugacy problem for nilpotent elements.

Much is known about (A) and (B). So we are left with problem (C). An obvious question is whether the number of conjugacy classes of nilpotent elements in $S$ is always finite. That this is not always the case was pointed out to the author by $\mathrm{L}$. Renner. His example is: $S=\left\{(\alpha A, \beta A) \mid \alpha, \beta \in K, A \in \mathscr{M}_{2}(K)\right\}$. We now prove the following general result.

THEOREM 4.2. Let $S$ be a connected regular monoid with zero and group of units $G$. Suppose that $S$ is Firreducible. If the number of conjugacy classes of nilpotent elements in $S$ is finite, then $G$ is nearly simple.

Proof. Let $J$ denote the nonzero minimum $\mathscr{J}$-class of $S$. Let $R$ denote the radical of $G$. Then $G=R G_{0}$ where $G_{0}$ is a semisimple group. By Theorem $1.3, \operatorname{dim} R=1$. Also $R$ is contained in the center of $G$. Suppose $G_{0}$ is not almost simple. Then by [8, Theorem 27.5], $G_{0}=G_{1} G_{2}$ where $G_{1}$ is a nontrivial semisimple subgroup of $G_{0}$ and $G_{2}$ is a nontrivial almost simple subgroup of $G_{0}$ and $G_{1}$ centralizes $G_{2}$. Let $S_{1}=\overline{R G_{1}}$ and let $J_{1}$ be a nonzero minimal $\mathscr{f}$ class of $S_{1}$. Since $R G_{1}$ is nearly semisimple, we see by [18, Theorem 2.3] that $J_{1}^{2} \nsubseteq J_{1}$. So there exists $a \in J_{1}$ such that $a^{2}=0$. Suppose $a G_{2} \subseteq S_{1} a S_{1}$. Then $G a G \subseteq S_{1} a S_{1}$ whereby $S a S=S_{1} a S_{1}$. So $J \subseteq S a S=J_{1} \cup\{0\}$. Thus $J=J_{1}$. Let $S_{2}=\overline{R G_{2}}$. Since $R G_{2}$ is nearly simple we see by [18, Theorem 2.3] that there exist nonzero minimal idempotents $e_{1}, e_{1}^{\prime}, e_{1} \neq e_{1}^{\prime}$ in the closure of a maximal torus of $R G_{2}$ such that $e_{1} \mathscr{J} e_{1}^{\prime}$ in $S_{2}$. Then $e_{1} e_{1}^{\prime}=0=e_{1}^{\prime} e_{1}$. Since $J$ is the minimum nonzero $\mathscr{J}$ class of $S$, there exists $e_{2} \in E(J)$ such that $e_{1} \geqslant e_{2}$. Now $e_{1}^{\prime}=x^{-1} e_{1} x$ for some $x \in R G_{2}$. Then since $e_{2} \in S_{1}, e_{1}^{\prime} \geqslant x^{-1} e_{2} x=e_{2}$. So $0=e_{1} e_{1}^{\prime}$ $\geqslant e_{2}$, a contradiction. Hence $a G_{2} \subseteq S_{1} a S_{1}$. Let

$$
V=\left\{g \in G_{2} \mid a g \in S_{1} a S_{1}\right\} .
$$

Clearly $V$ is a closed subset of $G_{2}$. Let $g, g^{\prime} \in V$. Then $a g, a g^{\prime} \in S_{1} a S_{1}$. So $a g g^{\prime} \in S_{1} a S_{1} g^{\prime}=S_{1} a g^{\prime} S_{1} \subseteq S_{1} a S_{1}$. Now let $x \in G_{2}, g \in V$. Then $a g \in S_{1} a S_{1}$ and $a x^{-1} g x=x^{-1} a g x \in x^{-1} S_{1} a S_{1} x=S_{1} a S_{1}$. Thus $V$ is a closed normal subgroup of $G_{2}$. By the above, $V \neq G_{2}$. Since $G_{2}$ is almost simple, $V$ is a finite subgroup of $G_{2}$ lying in the center of $G_{2}$. Let $|V|=m$. We can find a sequence of positive integers $n_{1}<n_{2}<\cdots, \alpha_{1}, \alpha_{2}, \ldots \in K^{*}$ such that the order of $\alpha_{i}$ is $n_{i}$ and $n_{i+1}>m n_{i}$, $i=1,2, \ldots$. Thus we can find $u_{1}, u_{2}, \ldots$ in a maximal torus of $G_{2}$ such that the order of $u_{i}$ is $n_{i}, i=1,2, \ldots$ Now $a u_{i}$ is a nilpotent element of $S$ for any $i \in Z^{+}$. Thus it suffices to show that $a u_{i}$ is not conjugate to $a u_{j}$ for $i<j$. So suppose to the contrary. Then there exists $x \in G$ such that $x^{-1} a u_{i} x=a u_{j}$. Now $x=y z$ for some $y \in R G_{1}$, $z \in G_{2}$. Then $a u_{i} y z=y z a u_{j}$. Thus $a y u_{i} z=y a z u_{j}$. So $a\left(z u_{j} z^{-1} u_{i}^{-1}\right)=y^{-1} a y \in S_{1} a S_{1}$ and $z u_{j} z^{-1} u_{i}^{-1} \in G_{2}$. Hence $z u_{j} z^{-1} u_{i}^{-1} \in V$ and $z u_{j} z^{-1} \in V u_{i}$. Since $u_{i}^{n_{i}}=1,|V|=m$, 
we see that $\left(z u_{j} z^{-1}\right)^{n_{i} m}=1$. Hence $u_{j}^{n_{i} m}=1$ and $n_{i} m \leqslant n_{j}$, a contradiction. This proves the theorem.

EXAmple 4.3. Theorem 4.2 shows that the Firreducible monoid $S=$ $\left\{A \otimes B \mid A, B \in \mathscr{M}_{2}(K)\right\}$ has infinitely many conjugacy classes of nilpotent elements.

EXample 4.4. Let $G_{0}=\left\{A \otimes\left(A^{-1}\right)^{t} \mid A \in \mathrm{SL}(3, K)\right\}, S=\overline{K G_{0}}, G=K^{*} G_{0}$. Then $G$ is nearly simple. Also $S$ is $\mathscr{J}$-irreducible. However the number of conjugacy classes of nilpotent elements in $S$ can be shown to be infinite.

ConjeCture 4.5. Let $S$ be a Firreducible connected regular monoid with zero. Then the number of conjugacy classes of nilpotent elements is finite if and only if $S$ is $\mathscr{J}$ linear.

Conjecture 4.6. Let $S$ be a connected $\mathscr{F}$ linear regular monoid with zero. Then the number of conjugacy classes of nilpotent elements in $S$ is equal to the number of conjugacy classes of nilpotent elements in the finite inverse monoid $\operatorname{Ren}(S)(\cong \operatorname{Munn}$ semigroup of $E(\bar{T})$ ).

REMARK 4.7. The Jordan canonical form shows the above conjecture to be true for $\mathscr{M}_{n}(K)$.

If $S$ is a regular semigroup with zero and if $a$ is a nilpotent element in $S$, then we say that $a$ is minimal if $a$ lies in a nonzero minimal $z$-class of $S$.

THEOREM 4.8. Let $S$ be a connected regular monoid with zero. Then the number of conjugacy classes of minimal nilpotent elements in $S$ equals the number of conjugacy classes of minimal nilpotent elements in $\operatorname{Ren}(S)$.

Proof. Let $T$ be a maximal torus of $G$. Let $X=\{(e, f) \mid e, f$ are nonzero minimal idempotents of $\bar{T}, e \neq f, e \mathscr{J} f\}$. Define an equivalence relation $\equiv$ on $X$ as follows: $(e, f) \equiv\left(e^{\prime}, f^{\prime}\right)$ if there exists $\theta \in W$ such that $e^{\theta}=e^{\prime}, f^{\theta}=f^{\prime}$. If $a$ is a minimal nilpotent element of $\operatorname{Ren}(S)$, then there exists a unique $(e, f) \in X$ such that $e \mathscr{R} a \mathscr{L} f$. It follows that the number of $\equiv$-classes of $X$ equals the number of conjugacy classes of minimal nilpotent elements in $\operatorname{Ren}(S)$. Now by Corollary 2.3, any minimal nilpotent element in $S$ is conjugate to an element of $e S f$ for some $(e, f) \in X$. Now let $(e, f) \in X, U=U(e, f)=e S f \backslash\{0\}$. Then every element of $U$ is a minimal nilpotent element and $U$ is an $\mathscr{H}$-class of $S$. Since $e S e$ is regular and $E(e S e)=\{0, e\}$ we see that $\operatorname{dim} e S e=1$. There exists $y \in G$ such that $f=y^{-1} e y$. Then $e S f=e S e y$. It follows that $\operatorname{dim} U=1$ and that $U$ is affine and irreducible. Now $T$ acts on $U$ by conjugation. By [8, Proposition 8.3] some orbit $A$ is closed in $U$. Suppose $\operatorname{dim} A=0$. Then $A=\{a\}, a \in C_{S}(T)=\bar{T}$ by [23, Theorem 4.4.4]. This is a contradiction since $a^{2}=0, a \neq 0$. Hence $\operatorname{dim} A=1$ and $A=U$. Thus any two elements in $U$ are conjugate. Now let $(e, f),\left(e^{\prime}, f^{\prime}\right) \in X, a \in U(e, f), b \in U\left(e^{\prime}, f^{\prime}\right)$ such that $a$ is conjugate to $b$. We must show that $(e, f) \equiv\left(e^{\prime}, f^{\prime}\right)$. Now $e \mathscr{R} a \mathscr{J} b \mathscr{R} e^{\prime}$. Hence by [16, Lemma 1.7], there exists $\sigma \in W$ such that $e^{\sigma}=e^{\prime}$. Thus without loss of generality we can assume that $e=e^{\prime}$. Now $b=x^{-1} a x$ for some $x \in G$. Then $x^{-1}$ ex $\mathscr{R} x^{-1} a x=b \mathscr{R}$ e. So $e \mathscr{R} x^{-1} e x$ and $x \in C_{G}^{r}(e)$. By [15, Theorem $1], a, b \in e S \subseteq S^{\prime}=\overline{C_{G}^{r}(e)}$. Now in $S, f^{\prime} \mathscr{L} b=x^{-1} a x \mathscr{L} x^{-1} f x$. Hence $f^{\prime} \mathscr{L} x^{-1} f x$ in $S$ and hence in $S^{\prime}$. Thus $f^{\prime} \mathscr{J} f$ in $S^{\prime}$. So by [16, Lemma 1.7] there exists 
$u \in N_{G}(T) \cap C_{G}^{r}(e)=N_{G}(T) \cap C_{G}(e)$ such that $f^{\prime}=u^{-1} f u$. Thus there exists $\theta \in W$ such that $e^{\theta}=e, f^{\theta}=f^{\prime}$. This proves the theorem.

Finally the author would like to thank Drs. T. E. Hall, K. S. S. Nambooripad, F. Pastijn and L. Renner for many useful discussions.

\section{REFERENCES}

1. A. H. Clifford and G. B. Preston, The algebraic theory of semigroups, vol. 1, Math. Surveys, no. 7 , Amer. Math. Soc., Providence, R.I., 1961.

2. R. J. H. Dawlings, Products of idempotents in the semigroup of singular endomorphisms of a finite dimensional vector space, Proc. Roy. Soc. Edinburgh Sect. A 91 (1981), 123-133.

3. __ On idempotent affine mappings, Proc. Roy. Soc. Edinburgh, Sect. A 93 (1983), 345-348.

4. J. A. Erdos, On products of idempotent matrices, Glasgow Math. J. 8 (1967), 118-122.

5. D. G. Fitz-Gerald, On the inverses of products of idempotents in regular semigroups, J. Austral. Math. Soc. 13 (1972), 335-337.

6. T. E. Hall, On regular semigroups, J. Algebra 24 (1973), 1-24.

7. J. M. Howie, An introduction to semigroup theorv, Academic Press, New York, 1976.

8. J. E. Humphreys, Linear algebraic groups, Springer-Verlag, Berlin and New York, 1981.

9. M. Loganathan, Cohomology and extensions of regular semigroups, J. Austral. Math. Soc. Ser. A 35 (1983), 178-193.

10. W. D. Munn, Pseudo-inverses in semigroups, Proc. Cambridge Philos. Soc. 57 (1961), 247-250.

11. K. S. S. Nambooripad, Structure of regular semigroups. I, Mem. Amer. Math. Soc. No. 22 (1979).

12. K. S. S. Nambooripad and F. Pastijn, V-regular semigroups, Proc. Roy. Soc. Edinburgh Sect. A 88 (1981), 275-291.

13. M. S. Putcha, On linear algebraic semigroups. II, Trans. Amer. Math. Soc. 259 (1980), 471-491.

14. On linear algebraic semigroups. III, Internat. J. Math. Math. Sci. 4 (1981), 667-690; Corrigendum 5 (1982), 205-207.

15. Green's relations on a connected algebraic monoid, Linear and Multilinear Algebra 12 (1982), 205-214.

16. Connected algebraic monoids, Trans. Amer. Math. Soc. 272 (1982), 693-709.

17. L_ Linear algebraic semigroups, Semigroup Forum 22 (1981), 287-309.

18. A semigroup approach to linear algebraic groups, J. Algebra 80 (1983), 164-185.

19. I I _ Idempotent cross-sections of $\mathbb{f}$ classes, Semigroup Forum 26 (1983), 103-109.

20. , A semigroup approach to linear algebraic groups. II. Roots, J. Pure Appl. Algebra (to appear).

21. Reductive groups and regular semigroups, Semigroup Forum 30 (1984), 253-261.

22. The group of units of a connected algebraic monoid, Linear and Multilinear Algebra 12 (1982), 37-50.

23. L. Renner, Algebraic monoids, Ph.D. Thesis, Univ. of British Columbia, 1982.

24. Reductive monoids are von-Neumann regular, J. Algebra (to appear).

25. Classification of semisimple rank one monoids, Trans. Amer. Math. Soc. 287 (1985), $457-493$.

26. Classification of semisimple algebraic monoids, Trans. Amer. Math. Soc. (to appear).

27. Analogue of the Bruhat decomposition for algebraic monoids, J. Algebra (to appear).

28. T. A. Springer, Linear algebraic groups, Birkhäuser, Basel, 1981.

29. R. Steinberg, Conjugacy classes in algebraic groups, Lecture Notes in Math., vol. 366, Springer-Verlag, Berlin and New York, 1974.

Department of Mathematics, North Carolina State University, Raleigh, North Carolina $27695-8205$ 